\title{
Connections between Intraparietal Sulcus and a Sensorimotor Network Underpin Sustained Tactile Attention
}

\author{
Dominique Goltz, ${ }^{1,2,3}$ @Christopher Gundlach, ${ }^{1,2}$ @Till Nierhaus, ${ }^{1,4,5}$ Arno Villringer, ${ }^{1,4,6}$ Matthias Müller, ${ }^{2}$ \\ and Burkhard Pleger ${ }^{1,6}$ \\ 'Department of Neurology, Max Planck Institute for Human Cognitive and Brain Sciences, 04103 Leipzig, Germany, ${ }^{2}$ Institute of Psychology, University of \\ Leipzig, 04103 Leipzig, Germany, ${ }^{3}$ Department of Systems Neuroscience, University Medical Center Hamburg-Eppendorf, 20246 Hamburg, Germany, \\ ${ }^{4}$ Berlin School of Mind and Brain, Mind and Brain Institute, 10099 Berlin, Germany, ${ }^{5}$ Neurocomputation and Neuroimaging Unit, Department of Education \\ and Psychology, Freie Universität Berlin, 14195 Berlin, Germany, and ${ }^{6}$ Clinic for Cognitive Neurology, University Hospital Leipzig, 04103 Leipzig, Germany
}

Previous studies on sustained tactile attention draw conclusions about underlying cortical networks by averaging over experimental conditions without considering attentional variance in single trials. This may have formed an imprecise picture of brain processes underpinning sustained tactile attention. In the present study, we simultaneously recorded EEG-fMRI and used modulations of steadystate somatosensory evoked potentials (SSSEPs) as a measure of attentional trial-by-trial variability. Therefore, frequency-tagged streams of vibrotactile stimulations were simultaneously presented to both index fingers. Human participants were cued to sustain attention to either the left or right finger stimulation and to press a button whenever they perceived a target pulse embedded in the to-be-attended stream. In-line with previous studies, a classical general linear model (GLM) analysis based on cued attention conditions revealed increased activity mainly in somatosensory and cerebellar regions. Yet, parametric modeling of the BOLD response using simultaneously recorded SSSEPs as a marker of attentional trial-by-trial variability quarried the intraparietal sulcus (IPS). The IPS in turn showed enhanced functional connectivity to a modality-unspecific attention network. However, this was only revealed on the basis of cued attention conditions in the classical GLM. By considering attentional variability as captured by SSSEPs, the IPS showed increased connectivity to a sensorimotor network, underpinning attentional selection processes between competing tactile stimuli and action choices (press a button or not). Thus, the current findings highlight the potential value by considering attentional variations in single trials and extend previous knowledge on the role of the IPS in tactile attention.

Key words: attention; brain connectivity; intraparietal sulcus; simultaneous EEG-fMRI; somatosensation; steady-state somatosensory evoked potentials

\section{Introduction}

The ability to continuously focus on relevant stimuli is a key feature of the human mind. This cognitive process, called sustained attention, has a tremendous influence on information processing and is a basic requirement for numerous skills, such as goal-directed behavior (Tipper et al., 1994). Since we are continuously faced with constantly competing sensory stimuli, an attentional selection mechanism seems mandatory. From studies in the visual domain it is known that the parietal cortex plays a crucial role in such attention processes (Behrmann et al., 2004). Specifically, it has been suggested that the superior parietal lobule

\footnotetext{
Received Aug. 13, 2014; revised March 30, 2015; accepted April 16, 2015.

Author contributions: D.G., A.V., M.M., and B.P. designed research; D.G., C.G., and T.N. performed research; D.G., C.G., T.N., A.V., and M.M. contributed unpublished reagents/analytic tools; D.G., C.G., and B.P. analyzed data; D.G. and B.P. wrote the paper.

This work was supported by the BMBF (Bernstein Focus, State Dependencies of Learning 01GQ0975), Project 18GL4DW4 awarded to B.P. We thank Klas Ihme and Norman Forschack for their help during data analyses and interpretation.

The authors declare no competing financial interests.

Correspondence should be addressed to Dominique Goltz, Department of Neurology, Max Planck Institute for Human Cognitive and Brain Sciences, Stephanstraße 1A, 04103 Leipzig, Germany. E-mail: goltz@cbs.mpg.de. DOI:10.1523/JNEUROSCI.3421-14.2015

Copyright $\odot 2015$ the authors $\quad 0270-6474 / 15 / 357938-12 \$ 15.00 / 0$
}

(SPL) subserves attentional shifts, whereas the intraparietal sulcus (IPS) guides the selection between competing stimuli (Molenberghs et al., 2007). A strengthened feedback connection between the IPS and extrastriate cortex (Gillebert et al., 2013) suggests that the IPS biases the competition between stimuli in upstream visual areas (Desimone and Duncan, 1995). Thus, it seems as if the IPS interacts with sensory regions to construct an attentional priority map and choose between competing visual stimuli.

In everyday life, not only visual but also tactile events catch our attention and sustained tactile attention is eminent for coordinating corresponding actions. Previous studies on tactile attention often investigated the role of lower sensory and higher cognitive brain regions; yet, similar interactions between the IPS and somatosensory regions have never been shown. Moreover, tactile attention research provides no hint that the activity in IPS could have a direct influence on behavioral performance. One reason may be that blocks of transient tactile stimuli were often presented with relatively long interstimulus intervals (Zopf et al., 2004; Galazky et al., 2009), so that there was no control over whether subjects constantly focused on the to-be-attended stimulus. Instead, cued attention conditions were compared with 
each other that might not reliably capture participants' actual attentional focus.

The aim of this study was to investigate brain regions involved in sustained tactile attention in more detail while better capturing participants' attentional variability. Because we were interested in brain regions involved in sustained attention and the competition between multiple stimuli, we presented continuous vibrotactile stimulation simultaneously to both index fingers and cued participants to attend to either the left (AL) or right (AR) stimulation. In EEG, frequency-tagged stimuli elicit steady-state somatosensory evoked potentials (SSSEPs) that can serve as a continuous measure of current attentional focus (Giabbiconi et al., 2007). On the one hand, we used these signals to investigate whether they are directly related to behavioral performance. On the other hand, we applied this EEG-marker of attention as a parametric modulator to simultaneously acquired fMRI data. First, we sought to replicate previous activity patterns in somatosensory-related regions by comparing cued attention conditions without accounting for attentional variability as revealed in dynamics of simultaneously recorded SSSEPs. Secondly, we employed parametric modeling using corresponding SSSEP responses to reveal attention effects based on participants' attentional trial-by-trial variability, as well as functional connectivity analyses to examine potential interactions within attentionrelated sensorimotor networks.

\section{Materials and Methods Participants}

Forty-seven volunteers between the ages of 21 and 34 (mean age $=26 \pm$ 3 years) were recruited as participants. Handedness was assessed by the Edinburgh Handedness Inventory (Oldfield, 1971) and only righthanded participants with a laterality quotient (LQ) $\geq 70$ were included (mean LQ $=93.6 \pm 9.2$ ). All participants reported normal or correctedto-normal vision, and no participant had a history of neurological or psychiatric disorder. The data of five participants were excluded from further analyses due to poor performance (4 participants: detection rate $<10 \%$, 1 participant had more incorrect than correct responses). The data of four additional participants could not be acquired due to technical problems with the EEG system. Thus, 38 participants (19 female; mean age $=26 \pm 2.66$ years, $\mathrm{LQ}=95.8 \pm 7.1$ ) remained in the sample for statistical testing. The study was approved by the Ethics Committee of the University of Leipzig, and participants gave written informed consent before the experiment.

\section{Stimuli and apparatus}

Vibrotactile trains of stimulations were simultaneously presented at participants' left and right index finger pad using two 8-dot piezo-electric stimulation displays ( 2 columns $\times 4$ rows pin matrix, $2.5 \mathrm{~mm}$ spacing; Piezostimulator, QuaeroSys; Fig. $1 A$ shows a schematic view of the stimulation device). The stimulation was driven by a constant $142 \mathrm{~Hz}$ carrier signal that was amplitude-modulated by 23.81 and $28.57 \mathrm{~Hz}$ for the left and right index fingers, respectively. Frequencies between 20 and $30 \mathrm{~Hz}$ were chosen, because this range was shown to cause the largest SSSEP amplitudes (Snyder, 1992). Note that two different frequencies were used for disentangling the electrophysiological responses from both fingers. Overall, the stimulations were perceived as ongoing vibrations of periodically changing intensities, lasting for $4.6 \mathrm{~s}$ ( $\pm 100 \mathrm{~ms}$ to reduce potential prediction of stimulus duration). As shown in Figure $1 B$, all pins moved simultaneously in an amplitude-modulated, sinusoidal fashion between 0 (all pins down) and $0.8 \mathrm{~mm}$ (all pins up). Occasionally, transient changes in stimulation amplitude were presented that felt like an additional stronger pulse. These pulses were variable increases in stimulation amplitude (pin height: $1.3-1.5 \mathrm{~mm}$ ) and lasted for $3.5 \mathrm{~ms}$. They appeared only on one pin column and participants had to discriminate whether these pulses were targets or distractors. If they occurred at the predefined target column (left or right pin column) at the to-be-attended stimulation (targets), participants were instructed to press a button with their right foot. If pulses occurred on the other column of the to-beattended stimulation or at any column of the to-be-ignored stimulation (distractors), participants were told not to press a button. The assignment of target column to left or right pin column was counterbalanced across participants and pseudorandomly assigned before the experiment. Targets and distractors were presented at stimulation valleys of the 23.81 or $28.57 \mathrm{~Hz}$ streams at pseudorandom points in time during a fixed stimulation window ( $200 \mathrm{~ms}$ after stimulation onset and $500 \mathrm{~ms}$ before stimulation offset). Maximally, they occurred twice within a trial with a minimal temporal interval of $1 \mathrm{~s}$ between each other. A trial incorporating a target or distractor is called an event-present trial (3/7 of all trials); all other trials were termed event-absent. The stimulation sequence was programmed with Presentation (v14.9, Neurobehavioral Systems).

\section{Procedure and design}

A trial started with a $500 \mathrm{~ms}$ presentation of a white arrow on a black screen that cued the to-be-attended stimulation for the upcoming trial (Fig. 1C). Simultaneously, vibrotactile stimulations were presented at participants' left and right index fingers, which lasted for $4.6 \pm 0.1 \mathrm{~s}$. Participants were asked to perform a discrimination task while fixating on a central white fixation cross $(4.1 \pm 0.1 \mathrm{~s})$. To this end, they had to continuously pay attention to the cued side and to press a button with their right foot as soon as they perceived a target pulse (i.e., stimulation increase in amplitude at one predefined target pin column). Due to the complex experimental setting, we instructed participants to focus on accuracy but to respond within a time window of $1 \mathrm{~s}$ after event presentation. The intertrial interval varied randomly between 3.65 and $5.15 \mathrm{~s}$. During this time a fixation cross was presented. Each participant was familiarized with the task before the experiment. The experiment consisted of 280 trials that were equally distributed over four experimental blocks. Trials were equally divided into AL finger and AR finger conditions. The order of trials was pseudorandomized with the constraint of no more than three successive trials of the same condition. Over all conditions, there were 160 event-absent trials. The remaining 120 trials included all possible conditions of target-distractor combinations.

\section{Simultaneous EEG-fMRI data acquisition}

Imaging was performed using a 3 T MRI System (Tim Trio, Siemens) equipped with a standard 12-channel head coil. BOLD (blood oxygen level-dependent)-sensitive images were collected using a $\mathrm{T} 2{ }^{*}$-weighted echoplanar imaging (EPI) sequence. For each of the four experimental blocks, we acquired 312 functional images in an interleaved mode comprising 30 slices covering the whole brain parallel to the anterior-posterior commissural plane [repetition time $(\mathrm{TR})=2000 \mathrm{~ms}$, echo time $(\mathrm{TE})=30 \mathrm{~ms}$, flip angle $=90^{\circ}$, image matrix $=64 \times 64$, field-of-view (FOV) of $19.2 \mathrm{~cm}$, spatial resolution $=3 \times 3 \times 4 \mathrm{~mm}$, interslice gap $=1$ $\mathrm{mm}]$. Anatomical images were recorded in a separate session using a T1-weighted 3D magnetization prepared rapid gradient echo $($ MP-RAGE $)$ sequence $\left(\mathrm{TR}=1300 \mathrm{~ms}, \mathrm{TE}=3.93 \mathrm{~ms}\right.$, flip angle $=10^{\circ}$, inversion time $=650 \mathrm{~ms}$, image matrix $=256 \times 240$, spatial resolution $=$ $1 \times 1 \times 1.5 \mathrm{~mm})$. A cushion was used to minimize participants' head motion, and before the experimental blocks, a field map measured the distortions of the magnetic field. To minimize artifacts in the EEG, the internal Tim Trio MRI ventilation system was disabled and the helium pump was switched off (Ritter and Villringer, 2006; Nierhaus et al., 2013).

Simultaneously with the fMRI, EEG recordings were acquired using an MRI-compatible EEG system consisting of an MR-compatible amplifier (BrainAmp MR, Brain Products) and an MR-compatible EEG-cap (BrainCap MR, Brain Products). Recordings from 31 scalp electrodes arranged according to the 10-20 system (FP1, FP2, F3, F4, C3, C4, P3, P4, O1, $\mathrm{O}_{2}, \mathrm{~F} 7, \mathrm{~F} 8, \mathrm{~T} 7, \mathrm{~T} 8, \mathrm{P} 7, \mathrm{P} 8, \mathrm{Fz}, \mathrm{Cz}, \mathrm{Pz}, \mathrm{Oz}, \mathrm{FC} 1, \mathrm{FC} 2, \mathrm{CP} 1, \mathrm{CP} 2$, FC5, FC6, CP5, CP6, TP9, TP10, POz) were obtained. Reference and ground electrodes were located at electrode position $\mathrm{FCz}$ and $\mathrm{AFz}$, respectively. An additional electrocardiogram channel (ECG) at participants' back was recorded for cardioballistic artifact removal. Impedance of all electrodes was kept $<5 \mathrm{k} \Omega$. EEG data were digitized at a sampling rate of 5 

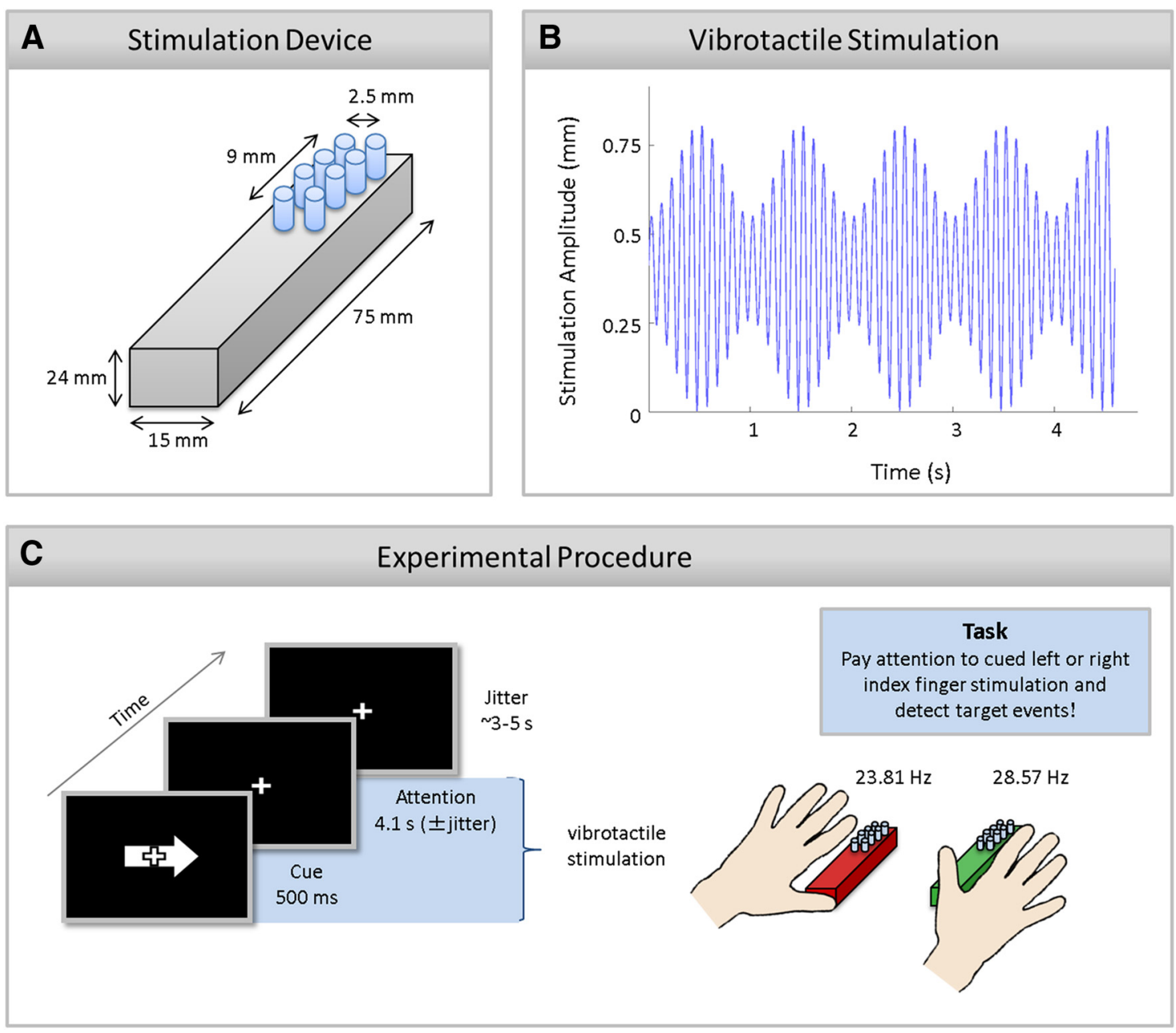

Figure 1. Schematic of the stimulation device $(\boldsymbol{A})$, vibrotactile stimulation stream $(\boldsymbol{B})$, and the experimental procedure $(\boldsymbol{C})$. $\boldsymbol{A}$, Lateral top view of the stimulation device for one finger. The stimulation display was composed of eight pins with 2 columns $\times 4$ rows. The diameter of one pin was $1.5 \mathrm{~mm}$ and the distance of two neighboring pins $2.5 \mathrm{~mm}$. $\boldsymbol{B}$, Schematic of tactile stimulation applied to the left and right index finger. A high-frequency carrier signal ( $142 \mathrm{~Hz}$, for display purposes $10 \mathrm{~Hz}$ are plotted) was amplitude-modulated by 23.81 and $28.57 \mathrm{~Hz}$ for left and right index finger, respectively (for display purposes $1 \mathrm{~Hz}$ is plotted). Note that an event-absent trial is depicted that was predominantly presented and that was used for all performed EEG and fMRI analyses. C, An exemplary trial in which participants were asked to pay attention to the right index finger stimulation. The visual arrow $(500 \mathrm{~ms})$ cued participants to allocate and sustain their attention to the left or right index finger stimulation. Participants were asked to detect a target event at the predefined target column on the to-be-attended stimulation. Note that stimulation devices are enlarged in this picture. The pin displays only covered a small part of the distal phalanges. The stimulation devices are depicted in red and green for a better comparison with Figures $2 B, C, 4$, and 6 .

$\mathrm{kHz}$ with an amplifier resolution of $0.5 \mu \mathrm{V}$ per bit and a $250 \mathrm{~Hz}$ low-pass filter. The EEG system was synchronized to the gradient-switching clock of the MR scanner using the Syncbox device (Brain Products). The amplifier and battery were seated inside the scanner bore to keep the cables that connect the amplifier with subjects' EEG cap as short as possible. The EEG equipment was immobilized using sandbags to minimize vibrations.

\section{Data analysis}

Behavioral data analysis

Behavioral responses were considered correct if a button press occurred within $1 \mathrm{~s}$ after target presentation. False alarms were defined as button presses within $1 \mathrm{~s}$ after distractor presentation. The percentage of correct responses (detection rate) and of false alarms was calculated. Moreover, reaction times were extracted. For each of these quantities, the values for the conditions $\mathrm{AL}$ and $\mathrm{AR}$ were compared by means of paired two-sided $t$ tests.

\section{EEG data analysis}

EEG data analysis was performed using MATLAB (MathWorks). First, a correction for gradient artifacts was performed using a self-built template-based subtraction method (Nierhaus et al., 2013). For this purpose, EEG data of each experimental block were segmented into epochs of $2 \mathrm{~s}$ (corresponding to the acquisition of each MRI volume, TR $=2 \mathrm{~s}$ ) and high-pass filtered (cutoff $55 \mathrm{~Hz}$ ). These filtered segments (mainly including scanner noise) were used to calculate the correlation of each segment with all other segments. Finally, a template for each segment was individually calculated and subtracted from the unfiltered data, by averaging 20 unfiltered segments that showed highest correlation for the filtered data. Subsequently, EEG data were low-pass filtered (cutoff 187.5 $\mathrm{Hz}$ ) and downsampled to $500 \mathrm{~Hz}$. Ballistocardiogram artifacts were detected and corrected by means of the AMRI toolbox (Liu et al., 2012). Noisy channels (impedances $\geq 10 \mathrm{k} \Omega$ ) and the ECG channel were removed from further analyses and EEG data were recalculated to average reference. As we were interested in trials with sustained allocation of attention without the processing and response to events, we only considered trials without any targets or distractors for EEG analysis. As SSSEPs are oscillatory responses that have the same frequency as the applied driving vibrotactile stimulus, they can easily be analyzed in the frequency domain (Morgan et al., 1996). Before transformation into frequency domain, detrending was performed to subtract any linear trend of each trial. SSSEP amplitudes were extracted by fast Fourier transformations (FFTs) from a time window of between 0.5 and $4.5 \mathrm{~s}$ after stimulus onset. The first $500 \mathrm{~ms}$ were discarded to exclude somatosensory evoked potentials to vibration onset. To identify appropriate electrodes for further 



Figure 2. Mean topographies and FFT-spectra for the left and right index finger stimulation. Only trials without any targets and distractors are included. $A$, An iso-contour voltage map was calculated based on the mean SSSEP amplitudes averaged across all participants and both experimental conditions (event-absent AL and AR). Electrodes with the strongest amplitudes are encircled and used for the subsequent analyses. $\boldsymbol{B}$, FFT-spectra with respect to the two applied stimulation frequency and the two conditions (AL and AR). Data of one participant are shown. Attending to the left (or right) stimulation frequency increases the respective SSSEP amplitude compared with attending to the right (or left) stimulation frequency. Note that for the left (or right) finger stimulation, attend right (or left) can serve as a baseline condition. We refer to this baseline condition also as ignore left (right). C, FFT-spectra with respect to the different experimental blocks. Exemplary data of one participant are shown. As variance across different experimental blocks is visible, percentage difference between single-trial SSSEP amplitudes and the respective block mean is derived.

analysis, an iso-contour voltage map was calculated based on the mean SSSEP amplitudes averaged across all participants and both experimental conditions (Gundlach and Müller, 2013). This was done for left and right stimulation separately. Figure $2 A$ depicts the grand mean topographical distributions. They show a typical EEG pattern observed for vibrotactile stimulation (cf. Severens et al., 2013; Pang and Mueller, 2014), as a dipole over the contralateral parietal cortex seems to project to frontal, as well as posterior parietal regions. Based on these mean topographies, a cluster of three electrodes showing the strongest amplitude was taken for subsequent analysis (Fig. 2A, circles). For the right index finger stimulation, $28.57 \mathrm{~Hz}$, the strongest amplitudes were at electrodes F3, Fz, and FC1 and for the left index finger stimulation, $23.81 \mathrm{~Hz}$, at Fz, F4, and FC2. Separately for both vibrotactile stimulations, we analyzed the frequency spectra of the two applied stimulation frequencies with respect to the two conditions (Fig. 2B). Given that the mean SSSEP amplitudes differed between experimental blocks (Fig. 2C), we corrected for any baseline differences by calculating the difference between single-trial SSSEP amplitude and block mean in percentage. Trials having SSSEP deflections larger than 3 SDs from experimental block average were not included in the subsequent fMRI analyses $(0.002 \%$ of all trials).

\section{Relationship between behavioral and EEG data}

To investigate whether attentional variability indexes behavioral performance, we directly related SSSEPs and behavioral hit rate and reaction times with each other. Note that event trials (targets and distractors) and subsequent button presses do not allow a reliable analysis of steady-state responses, which is why we were primarily interested in whether SSSEPs index previous or upcoming behavioral performance. To this end, we grouped outlier-corrected SSSEP responses in previous $(n-1)$ or upcoming $(n+1)$ event-absent trials based on whether participants correctly detected (hit) or missed to respond to the target (miss) presented at the $n^{\text {th }}$ trial. In $10.13 \%$ of all trials, event-absent trials preceded target trials and in $9.52 \%$ event-absent trials followed them. For investigating the relationship between hit rates and preceding or upcoming SSSEP amplitudes, we averaged SSSEP amplitudes for hits and misses and performed, separately for both conditions $(n-1$ and $n+1)$, paired sample $t$ tests between corresponding SSSEPs. Given that we entered the same
SSSEP values in these analyses as in the EEG-informed general linear model (GLM; i.e., deviation from block mean), one-sample $t$ tests were additionally performed to test whether SSSEP values for hits and misses significantly differ from zero (block mean). For analyzing the relationship between reaction times and SSSEPs, we decided to further increase the limited number of trials by also including reaction times slower than $1 \mathrm{~s}$ and only analyzed correctly detected target trials (8.5\% of all trials). For each participant and separately for both conditions $(n-1$ and $n+$ 1), we averaged SSSEP amplitudes and reaction times and subjected them to correlation analyses using Pearson's $r$.

\section{fMRI data analysis}

MRI data were analyzed using SPM 8 (Wellcome Trust Centre for Neuroimaging, UCL, London, UK). Functional volumes were slice timecorrected using the middle slice as reference, spatially realigned to the first image of the time series using a six-parameter rigid body transformation. To account for magnetic field inhomogeneities, a distortion correction based on the field map measurement was applied. Thereafter, functional and anatomical images were coregistered. Anatomical images were segmented into gray and white matter, as well as CSF, and normalized to a standard stereotaxic space using the T1 template by the Montreal Neurological Institute (MNI) delivered with SPM. The normalization parameters were then applied to the functional EPI series. Spatial smoothing was performed on the functional data using a threedimensional Gaussian filter of $8 \mathrm{~mm}$ full-width at half-maximum. Given that parametric regressors change the explained variance of the main regressors we used separate GLMs for the cued attention effects (conventional GLM) and attention effects based on attentional trial-by-trial variability (EEG-informed GLM). For the EEG-informed analysis, we calculated two GLMs, one for each stimulation frequency; that means that we compared both attention conditions as a function of stimulation frequency (compare Fig. $2 B$ ). As a consequence, the condition in which participants attended to the other stimulation frequency could serve as an ignore (or distraction) condition. Note that we chose to calculate two instead of one combined EEG-informed GLM analysis, because the parametric regressors were not completely orthogonal to each other. Additionally, we wanted to prevent an additional decrease of analyzable trials 
because the two electrode clusters (that were used for the different stimulation frequencies) were noisy in different trials.

Classical GLM based on cued attention conditions. To examine BOLD effects of cued attention conditions, we applied voxelwise statistical analyses using the GLM. Five regressors were specified for GLM: (1) eventabsent trials in which participants were asked to pay attention to left finger stimulation $(\mathrm{AL}),(2)$ event-absent trials in which participants were asked to pay attention to right finger stimulation (AR), (3) eventpresent trials for $\mathrm{AL},(4)$ event-present trials for $\mathrm{AR}$, and (5) button presses. Because target trials were heavily confounded by subsequent motor movements (i.e., press a button) and we were not interested in the processing of targets and distractors, we only focused on the effects of event-absent AL and event-absent AR, and we compared these two active tactile attention conditions with each other. Note that $\mathrm{AL}$ can also be considered as ignore right finger (IR) and vice versa. Predictors of the hemodynamic response were modeled by a stick function, placed at the onset of the vibrotactile stimulation. The stick function was convolved with a canonical hemodynamic response function (event-related analysis). A temporal high-pass filter of $100 \mathrm{~s}$ was used to remove lowfrequency drifts. We performed random-effects analyses by first contrasting event-absent $\mathrm{AL}$ with event-absent $\mathrm{AR}$ and vice versa on a single-subject level. These individual contrast images were then entered into a second-level group analysis using one-sample $t$ tests. The resulting $t$ maps were thresholded at $p<0.001$ uncorrected, with the extent threshold of $k \geq 10$ as cluster size. Clusters of BOLD activity surpassing a threshold of $p<0.05$ (FWE-corrected; cluster-level) were considered as significant. All reported coordinates correspond to the anatomical MNI space as used in SPM 8. The probability maps of the Anatomy Toolbox (v1.7; Eickhoff et al., 2007) were used for assignment of BOLD responses to their underlying Brodmann areas. Maximal activation refers to voxels with the highest statistical $t$ value in the respective brain region. For illustration purposes, functional activation maps thresholded at $p<0.001$ (uncorrected; $k \geq 10$ ) were superimposed on an anatomical template (ch2better.nii.gz) as provided in MRIcroN (v6; www.mccauslandcenter.sc.edu/mricro/mricron/).

Parametric modeling of FMRI data using SSSEPs as a marker of attentional trial-by-trial variability. To identify those brain regions within the fMRI data that directly relate to the attentional variability as captured with EEG, we examined neural differences between the two task conditions (i.e., event-absent trials in AL and AR) in relation to the SSSEP marker of attention. To this end, we entered the SSSEP amplitude differences (i.e., percentage deviation of single-trial amplitude from block mean SSSEP, see "EEG data analysis", above, for further details) as trialby-trial parametric modulations of the respective fMRI scans in the GLM. Because each stimulation was either attended or ignored (i.e., attend to other stimulation), we calculated two separate GLMs, one for the left and the other for the right finger stimulation (compare Fig. 2B). Accordingly, for the left finger stimulation, we set up the following GLM: (1) event-absent AL, (2) parametric modulator: event-absent AL SSSEPs for $23.81 \mathrm{~Hz}$ as captured by EEG electrode cluster over the right hemisphere (ALpar), (3) event-absent ignore left finger (IL), (4) parametric modulator: event-absent IL SSSEPs for $23.81 \mathrm{~Hz}$ as captured by EEG electrode cluster over the right hemisphere (ILpar), (5) event-present AL, (6) event-present IL, and (7) button presses. For the right finger stimulation, we set up the following GLM: (1) event-absent AR, (2) parametric modulator: event-absent AR SSSEPs for $28.57 \mathrm{~Hz}$ from EEG electrodes over the left hemisphere (ARpar), (3) event-absent IR, (4) parametric modulator: event-absent IR SSSEPs for $28.57 \mathrm{~Hz}$ from EEG electrodes over the left hemisphere (IRpar), (5) event-present AR, (6) event-present IR, and (7) button presses. On a single-subject level, we compared the SSSEP-based parametric modulators of the attend and ignore conditions. But note that because IL is equivalent to the AR condition for 23.81 $\mathrm{Hz}$ and IR to AL for $28.57 \mathrm{~Hz}$ (compare Fig. $2 B$ ), we always directly compared the parametric modulators of left and right attention conditions. The individual contrast images were then entered into a secondlevel analysis using one-sample $t$ tests. The significance level for the analysis was set to $p<0.001$ (uncorrected) with an extent threshold $k \geq$ 10 as cluster size. For directly visualizing that the BOLD signal scaled with the amplitude of the simultaneously recorded SSSEPs, we extracted the first eigenvariate from those areas that correlated with the SSSEP markers of attention and clustered them according to equally sized low, medium, and high SSSEP amplitude bins. Finally, to investigate whether these areas are also involved in sustained attention or whether they are only involved in controlling the trial-by-trial variations thereof, we calculated the respective contrast estimates in the classical GLM.

Psychophysiological interactions reflecting functional connectivity based on classical GLM versus SSSEP-informed GLM. To assess task-related functional connectivity of those areas that correlated with the trial-bytrial SSSEP markers of attention, we applied the method of psychophysiological interactions (PPIs; Friston et al., 1997). Clusters of brain areas that correlated with the SSSEP-informed attention effects on the group level were chosen as seed regions (see "Parametric modeling of fMRI data using SSSEPs as a marker of attentional trial-by-trial variability", above, on how we identified those brain areas). On the single-subject level, we extracted the first eigenvariate of these seed regions and created the respective psychophysiological interaction term. The PPI term was based either on cued attention conditions (i.e., main regressors in the conventional GLM) or the attention effect as captured by the SSSEP (i.e., parametric regressors in parametric GLM). Both PPI terms, together with their corresponding $\mathrm{Y}$ and $\mathrm{P}$ regressors, were separately entered into individual subject GLMs (conventional or parametric). Within the GLM, the $\mathrm{Y}$ regressor represents the BOLD activity obtained from the region of interest and the $\mathrm{P}$ regressor represents the task-related variance. We added both regressors to the PPI GLM to guarantee that the PPI term exclusively addresses the interaction between the task, BOLD activity, and, only for the EEG-informed PPI, the SSSEPs. The resulting PPI contrast images were then subjected to group-level analysis using onesample $t$ tests. The significance level for the PPI analysis was set to $p<$ 0.005 (uncorrected) with an extent threshold $k \geq 10$ as cluster size.

\section{Results}

\section{Behavioral data}

Across all participants and conditions, $68.5 \%$ of targets were detected with a mean false alarm rate of $0.15 \%$ and a mean reaction time of $0.7 \mathrm{~s}$. The mean detection rate for $\mathrm{AL}$ was $65 \%$ (SE = $3.5 \%)$ with a false alarm rate of $0.16 \%(\mathrm{SE}=0.01 \%)$. The mean reaction time was $0.71 \mathrm{~s}(\mathrm{SE}=0.01 \mathrm{~s})$. For $\mathrm{AR}, 72 \%(\mathrm{SE}=2.8 \%)$ of target events were detected with a mean reaction time of $0.69 \mathrm{~s}$ $(\mathrm{SE}=0.01 \mathrm{~s})$. Participants pressed incorrectly in $0.14 \%$ ( $\mathrm{SE}=$ $0.02 \%)$ of distractor events. There was a significant difference between left and right attention conditions in detection rate $\left(t_{(37)}=2.57, p<0.05\right)$ but not for false alarms $\left(t_{(37)}=1.39, p=\right.$ $0.17)$ or reaction times $\left(t_{(37)}=1.59, p=0.12\right)$.

\section{Relationship between behavioral and EEG data}

To investigate whether attentional variability indexes behavioral performance, we directly related hit rates and reaction times in target trials with preceding and following SSSEPs of event-absent trials. For the condition in which event-absent trials preceded a target trial $(n-1)$, there was a significant difference in the preceding SSSEP values for hits and misses $\left(t_{(37)}=2.28, p<0.05\right)$. As shown in Figure $3 A$, steady-state amplitudes are significantly lower before missed target events (SSSEP $=-7.92 \%$ deviation from block mean, $\mathrm{SE}=2.9 \%$ ) than before hits $(\mathrm{SSSEP}=-1.06 \%$ deviation from block mean, $\mathrm{SE}=1.5 \%$ ). This is corroborated by the fact that SSSEP amplitudes before misses are significantly lower than block mean $\left(t_{(37)}=-2.75, p<0.01\right)$ but not SSSEP amplitudes previous to hit trials $\left(t_{(37)}=-0.71, p=0.49\right)$. Moreover, as shown in Figure $3 B$, there was a significant negative correlation between reaction times at target trials and preceding SSSEP values ( $r=-0.37, p<0.05)$. That means, the higher the steady-state amplitudes the lower was the reaction times in subsequent target trials. No effects were found between behavioral performance (at $n^{\text {th }}$ trial) and subsequent steady-state responses $(n+1$ trial $)$. 


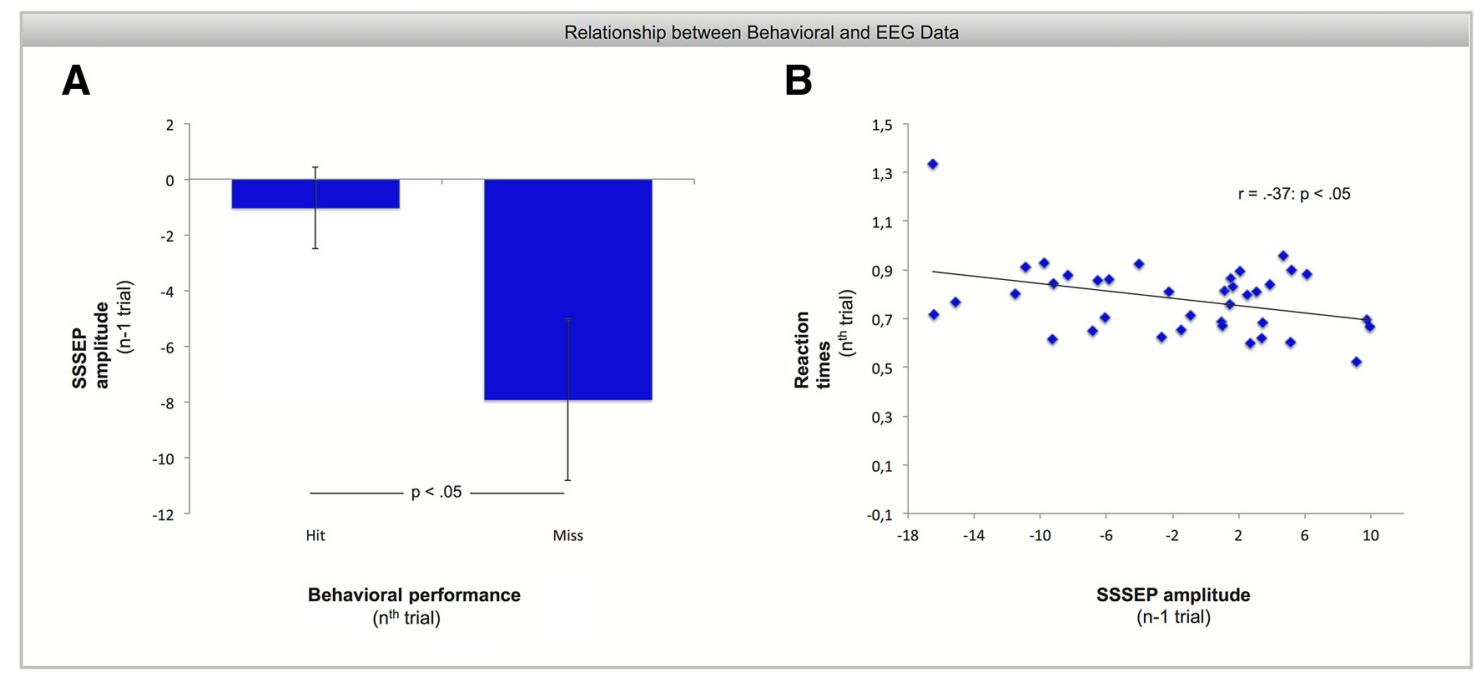

Figure 3. Relationship between behavioral and steady-state responses. Note that SSSEP amplitudes refer to the difference from blockmean (in percentage). Error bars indicate the SEM. $A$, Lower steady-state amplitudes cause significantly more missed target events in the subsequent trial than steady-state responses close to block mean $\left(t_{(37)}=2.28, p<0.05\right)$. Note that this analysis was based only on $10.13 \%$ of all trials and that steady-state amplitudes before hits do not necessarily have to be higher than block mean only because steady-state responses before missed targets are significantly lower than block mean. There is still a large amount on event-absent trials that were presented subsequently after each other or before distractor trials that are not included in the analyses. $\boldsymbol{B}$, There is a significant correlation between steady-state responses and reaction times. The higher the steady-state amplitude in the previous trial, the lower was the reaction time to correctly detected target events in subsequent trials.

Table 1. Anatomical locations and statistical information on all activated clusters for classical GLM and parametrical modeling analyses

\begin{tabular}{|c|c|c|c|c|c|c|c|}
\hline Contrast & Anatomical region & Area & Cluster size & Peak level $t$ value & $x$ & $y$ & $z$ \\
\hline \multirow[t]{9}{*}{$A L>A R$} & \multirow[t]{2}{*}{ Right SI* } & 1 & 1835 (744) & 9.71 & 51 & -19 & 49 \\
\hline & & $3 b$ & 1835 (744) & 7.53 & 27 & -37 & 49 \\
\hline & Right SII* & $\mathrm{OP} 3$ & $1835(24)$ & 8.14 & 45 & -19 & 22 \\
\hline & Right MI* & $4 a$ & 1835 (744) & 8.37 & 39 & -16 & 58 \\
\hline & Right SPL* & SPL (7PC) & 1835 (744) & 6.75 & 36 & -49 & 64 \\
\hline & Right putamen & & 1835 & 5.49 & 30 & -7 & 1 \\
\hline & Right thalamus & Thal-parietal & 87 & 5.43 & 15 & -19 & 10 \\
\hline & \multirow[t]{2}{*}{ Left cerebellum* } & Lobule VI & $125(29)$ & 6.85 & -24 & -52 & -26 \\
\hline & & Lobule VIIllb & 29 & 5.28 & -18 & -61 & -47 \\
\hline \multirow[t]{13}{*}{$A R>A L$} & \multirow[t]{2}{*}{ Left SI* } & 2 & $2252(1082)$ & 12.07 & -45 & -34 & 52 \\
\hline & & $3 b$ & 21 & 5.50 & 66 & -4 & 16 \\
\hline & \multirow[t]{2}{*}{ Left SII } & OP 1 & 2252 (1082) & 5.93 & -48 & -22 & 22 \\
\hline & & $\mathrm{OP} 3$ & $2252(1082)$ & 5.84 & -51 & -16 & 19 \\
\hline & Left SPL* & SPL (7PC) & 2252 (1082) & 8.98 & -30 & -49 & 64 \\
\hline & Left IPL* & PFt & 2252 (1082) & 5.78 & -57 & -19 & 28 \\
\hline & Left precentral gyrus & 44 & 64 & 5.38 & -57 & 8 & 37 \\
\hline & Left temporal pole & TE 3 & 13 & 3.74 & -51 & 8 & -8 \\
\hline & Left calcarine sulcus & h0c1 (V1) & 18 & 4.01 & -6 & -91 & 7 \\
\hline & Right lingual gyrus & h0C3v (V3v) & 12 & 3.96 & 24 & -85 & -5 \\
\hline & Left putamen & & 2252 & 4.83 & -30 & -4 & -5 \\
\hline & \multirow[t]{2}{*}{ Right cerebellum* } & Lobule VI & $286(65)$ & 7.53 & 24 & -52 & -26 \\
\hline & & Lobule VIIla & $50(10)$ & 6.30 & 18 & -64 & -44 \\
\hline \multirow{2}{*}{ ARpar $>$ IRpar } & Right IPS & hIP2 & 68 & 4.42 & 48 & -43 & 40 \\
\hline & Left IPS & hIP1 & 16 & 4.17 & -39 & -55 & 40 \\
\hline
\end{tabular}

Listed are all activated brain regions that showed increased activity in the corresponding contrast $(p<0.001$, cluster size $k \geq 10)$. Coordinates are in MNI space. IPL, inferior parietal lobe.

*Denotes brain regions that surpassed a threshold of $p<0.05$ (FWE-corrected; cluster-level). The corresponding cluster size according to the FWE-correction is in brackets.

\section{fMRI data}

To identify brain regions that are involved in tactile spatial attention, we compared event-absent AL and AR with each other. First, this was done by means of a conventional GLM based on cued attention conditions. In a second step, we used SSSEPs as marker of attentional trial-by-trial variability. Accordingly, we performed an additional analysis in which the EEG signal served as a parametric modulator of the respective main regressors, event-absent AL and AR. These EEG parametric regressors were then compared against each other. A summary of all activated areas is shown in Table 1.

\section{Classical GLM based on cued attention conditions}

When participants were asked to pay attention to their left compared with right index finger $(\mathrm{AL}>\mathrm{AR})$, we found three significant activation clusters. One activation cluster was mainly covering the right postcentral gyrus, SI, but also extended more rostrally to primary motor cortex (MI), as well as more caudally 


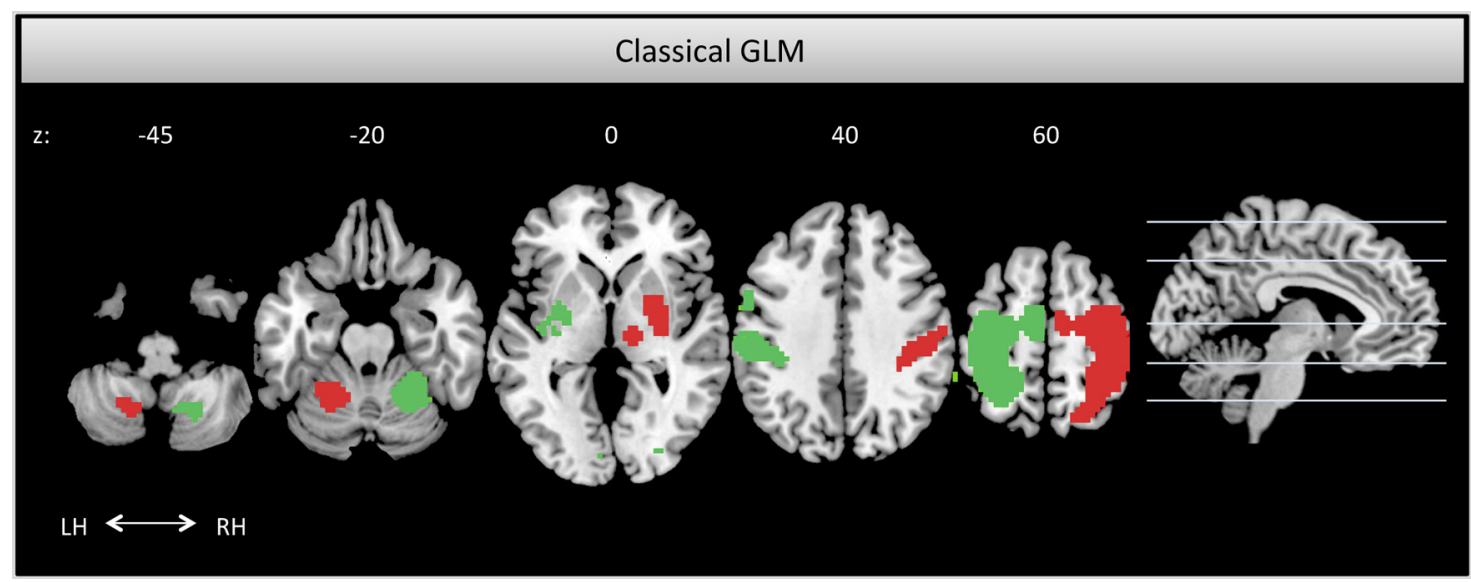

Figure 4. Horizontal activation maps for the comparisons event-absent AL $>A R$ in red and event-absent AR $>$ AL in green, thresholded at $p<0.001$ uncorrected. Both contrasts revealed increased activity in the contralateral primary and secondary somatosensory cortices, as well as in motor areas and ipsilateral cerebellar regions. Abbreviation for the left and right hemisphere is $\mathrm{LH}$ and RH, respectively, and the respective $z$-coordinates are in MNI space.

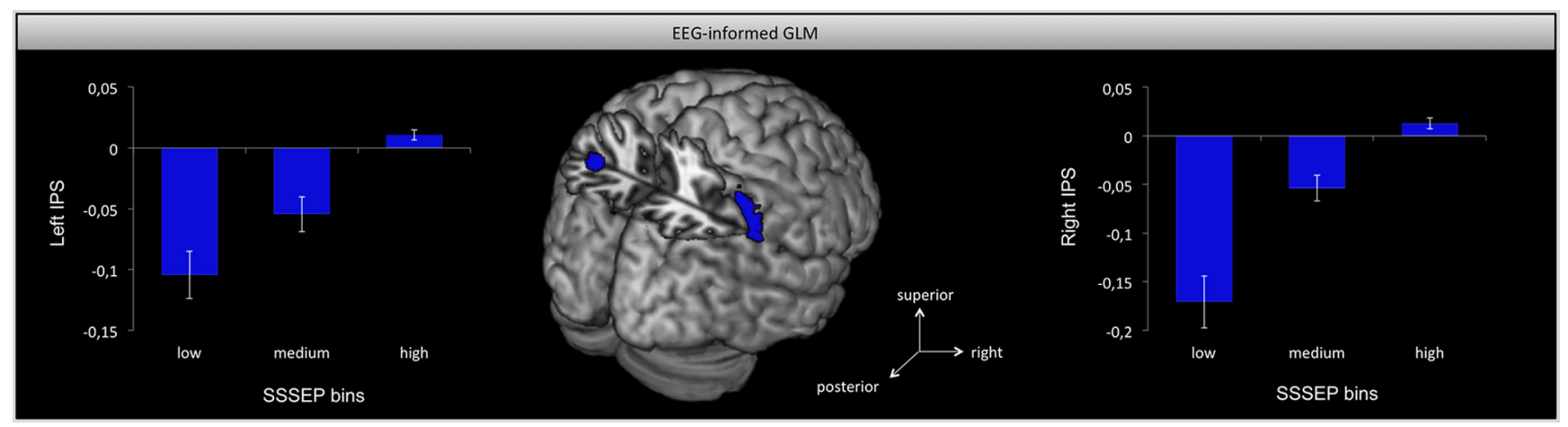

Figure 5. Lateral top view of the left and right IPS activation for the comparison event-absent ARpar $>$ IRpar, thresholded at $p<0.001$ uncorrected, revealed by EEG-informed fMRI analysis including SSSEPs as parametric modulator. The bar graphs on the left and right display normalized contrast estimates for the parametric modulation in left and right IPS. Error bars indicate the SEM. The figure shows that activity in the IPS scaled with the amplitude of the steady-state responses; i.e., the higher the steady-state amplitude, the higher the IPS BOLD activity. Note that we have to be cautious with the reference value because we did not assess a reliable baseline activity.

to superior parietal regions. Additionally, increased activity was revealed in the right secondary somatosensory cortex (SII), as well as in left cerebellar regions (mainly covering and peaking in lobule VI). Statistical parametric maps depicting the activated regions are illustrated in Figure 4, red. When participants were instructed to pay attention to their right index finger (contrast AR > AL; Fig. 4, green), left SI and SII showed increased activity. This cluster also expanded to surrounding areas, such as superior, as well as inferior, parietal cortex. Furthermore, increased activity was revealed in right cerebellar regions (lobules VI and VIIIa).

Parametric modeling of fMRI data using SSSEPs as a marker of attentional trial-by-trial variability

Investigating the attention effects based on the SSSEP parameter, we found an increased activity in the right and left inferior parietal cortex both with maximal activity in IPS (hIP2 and hIP1, respectively). However, this was only revealed for the right finger stimulation (ARpar > IRpar; Fig. 5). For the left finger, activation in right IPS was revealed only by trend (ALpar $>$ ILpar; $p<0.01$, uncorrected). The parametric modulation in the right and left IPS for the right finger stimulation is especially illustrated in the bar graphs of Figure 5. But note that although the bar graphs suppose negative BOLD responses for low and medium SSSEP bins and positive BOLD responses for high bins, we cannot refer to the BOLD activity as positive and negative, because we did not include any BOLD baseline measurements (such as, tactile stimulation without attention, null trials) in our experimental design. Nevertheless, the figure clearly shows that the activity in left and right intraparietal sulcus scaled with the amplitude of the SSSEP signal, i.e., the intraparietal sulcus activity increases with increasing SSSEP amplitudes.

A direct comparison between the classical GLM and the EEGinformed findings is shown in Figure 6A. This brain picture suggests that although somatosensory cortices are involved in sustained attention (as captured with the classical GLM), the IPS is primarily involved in controlling the trial-by-trial variations thereof. To substantiate this, we calculated the respective contrast estimates of the IPS in the classical GLM approach. As shown in the bar graphs of Figure $6 B$, activity of the left and right IPS was not significantly activated in the sustained attention conditions (classical GLM).

\section{PPIs reflecting functional connectivity based on classical GLM versus SSSEP-informed GLM}

Table 2 summarizes all calculated PPIs with the corresponding results. Because left and right horizontal intraparietal sulci are anatomically detached and previous findings suggest different response patterns in an attention task (Vandenberghe et al., 2005), we performed separate PPIs for the two IPS clusters. When participants were cued to pay attention to their right finger 


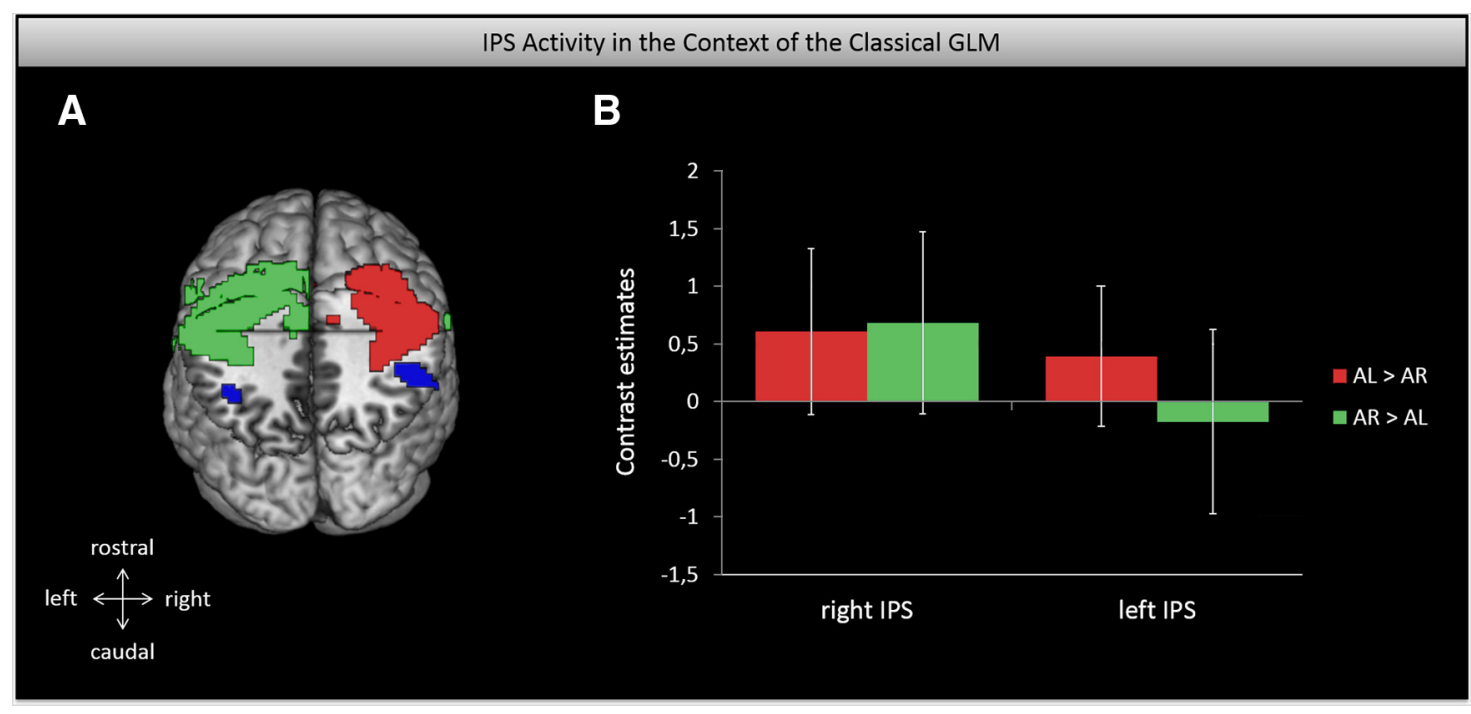

Figure 6. The IPS in the context of the classical GLM approach. $A$, Caudal top view of the IPS and somatosensory cortex activation as captured with the EEG-informed and the classical GLM analyses, respectively. Both thresholded at $p<0.001$ uncorrected. $\boldsymbol{B}$, Bar graphs display normalized contrast estimates for the left and right IPS in the classical GLM contrasts, AL versus AR and AR versus AL. Error bars show $90 \%$ confidence intervals. It seems as if the IPS is not primarily involved in sustained attention (as captured with classical GLM), but rather in the trial-by-trial variations thereof.

$(\mathrm{AR}>\mathrm{AL}$, conventional GLM), we found an increased functional coupling between right IPS and several distributed areas including inferior parietal lobe, frontal gyri, visual areas, and cerebellar as well as amygdala regions that also extended into hippocampal regions (Fig. 7). In contrast, when accounting for the attention effects based on the SSSEP signal (ARpar > IRpar), which is a measure of attentional trial-by-trial variability, we found an increased coupling to primary, as well as secondary, somatosensory cortices (Fig. 8). Moreover, increased connectivity was found to other key regions involved in sensorimotor processing such as premotor and primary motor regions, caudate nucleus, superior frontal regions, and thalamic regions. Considering the left horizontal IPS with respect to cued attention conditions based on the conventional GLM (AR > AL), we found increased connectivity to surrounding inferior parts of the left parietal cortex, as well as to the superior frontal gyrus, cerebellar regions, thalamus, visual areas, and amygdala extending to hippocampus. In contrast, including SSSEP signals as parametric modulators (ARpar > IRpar), we found an increased coupling between left IPS and left SII, MI, right inferior parietal lobe, and right superior occipital gyrus.

\section{Discussion}

The study aimed to investigate sustained tactile attention while having control over participants' attentional focus over time. Given that modulations of SSSEPs are reliable markers of tactile attentional allocation (Giabbiconi et al., 2004; Pang and Mueller, 2014), we recorded simultaneous EEG-fMRI and asked participants to continuously pay attention to either left or right index finger which were both simultaneously stimulated by vibrotactile streams. This allowed us to continuously measure each individual's attentional trial-by-trial variability and to apply corresponding SSSEP markers to fMRI data analyses for a reliable assessment of attention-modulating brain regions and their embedment in brain networks of sensorimotor integration.

Conventional GLM analyses comparing cued attention conditions against each other revealed attentional modulations in contralateral primary and secondary somatosensory cortices, as well as in ipsilateral cerebellar cortices, which is well in-line with previous research on tactile attention (Johansen-Berg et al., 2000;
Burton et al., 2008; Goltz et al., 2014). Parametric modulation analyses using SSSEPs as a marker of attentional trial-by-trial variability exposed activity in bilateral IPS, in particular when attention was paid to the right index finger (i.e., dominant hand). In this context, functional connectivity analyses showed an increased functional coupling between right IPS and somatosensory, as well as (pre)motor cortices and caudate nucleus (SSSEPinformed GLM; Fig. 8). This is in contrast to the increased functional connectivity pattern that we found based on cued attention conditions alone (classical GLM; Fig. 7). This analysis revealed an increased coupling to a widely distributed network including frontal, parietal, occipital, cerebellar, and limbic areas. Although this attention network is task-related, it seems to have no specific connections to the task-relevant somatosensory cortices.

These striking differences between the classical and the EEGinformed analysis are most likely due to different aspects of endogenous attention that these two analyses capture. Whereas the classical analysis reveals attention effects based on external attentional cues (i.e., visual arrows), the EEG-informed analysis addresses attentional trial-by-trial variability. Given that this variability does not correlate with our experimental conditions and differed considerably between subjects, this attention effect seems to be intrinsically driven. In light of the present findings, considering modulations of SSSEPs as markers of single-trial neuronal states seems advantageous to improve temporal resolution of fMRI important for capturing intrinsically driven, fast fluctuating cognitive states and associated network function. Thus, simultaneous EEG-fMRI bears huge potential to promote brain research on sustained attention, not only in the tactile but presumably also in the auditory and visual domain.

The IPS plays a major role in the analyses because it correlated with attention-modulated SSSEP amplitudes. Given that the task required the purposeful allocation of attention to predetermined locations in space, our finding seems to be in-line with studies showing that the IPS is involved in endogenous attentional orienting (Corbetta and Shulman, 2002; Mayer et al., 2004). However, the current study is the first showing that the IPS explicitly contributes to attentional variability over time in such top-down 
Table 2. Anatomical locations and statistical information on all activated clusters for PPI analyses

\begin{tabular}{|c|c|c|c|c|c|c|c|c|}
\hline Seed region & Contrast & Anatomical region & Area & Cluster size & Peak level $t$ value & $x$ & $y$ & $z$ \\
\hline \multirow{20}{*}{ Right IPS } & \multirow[t]{20}{*}{$A R>A L$} & Left IPL & $\mathrm{PFm}$ & 447 & 4.68 & -48 & -49 & 25 \\
\hline & & Right IPL & PGa & 10 & 3.28 & 48 & -58 & 34 \\
\hline & & \multirow[t]{2}{*}{ Left superior frontal gyrus } & \multirow[t]{2}{*}{$\mathrm{Fp} 1$} & 305 & 4.96 & -24 & 56 & 25 \\
\hline & & & & 64 & 3.69 & -18 & 26 & 61 \\
\hline & & Left middle frontal gyrus & 45 & 134 & 6.09 & -48 & 23 & 40 \\
\hline & & Left inferior frontal gyrus & 45 & 82 & 3.80 & -48 & 23 & 4 \\
\hline & & Right inferior frontal gyrus & 45 & 10 & 2.94 & 51 & 26 & 4 \\
\hline & & \multirow[t]{2}{*}{ Left middle temporal gyrus } & \multirow[t]{2}{*}{ PGa } & 447 & 4.24 & -48 & -58 & 19 \\
\hline & & & & 33 & 3.36 & -57 & -19 & -5 \\
\hline & & Right superior temporal gyrus & TE 1.0 & 22 & 3.57 & 51 & -22 & 7 \\
\hline & & Right middle temporal gyrus & h0v5 (V5) & 10 & 3.03 & 54 & -64 & 7 \\
\hline & & Left cuneus & $\mathrm{h} 0 \mathrm{c} 4 \mathrm{~d}(\mathrm{~V} 3 \mathrm{~A})$ & 12 & 3.15 & -12 & -85 & 16 \\
\hline & & Right cuneus & h0c4d (V3A) & 88 & 3.45 & 15 & -79 & 22 \\
\hline & & Right fusiform gyrus & FG1 & 78 & 4.32 & 39 & -58 & -14 \\
\hline & & Right amygdala & $\mathrm{LB}$ & 31 & 3.95 & 33 & -7 & -17 \\
\hline & & Left amygdala & $C M$ & 12 & 3.01 & -24 & -7 & -14 \\
\hline & & \multirow[t]{3}{*}{ Left cerebellum } & Lobule V & 119 & 3.78 & -9 & -52 & -14 \\
\hline & & & Lobule VIla & 63 & 3.40 & -30 & -79 & -29 \\
\hline & & & IX & 23 & 2.88 & -6 & -61 & -41 \\
\hline & & Right cerebellum & Lobule VIla & 34 & 3.82 & 30 & -67 & -35 \\
\hline \multirow[t]{8}{*}{ Left IPS } & \multirow[t]{8}{*}{$A R>A L$} & Left IPL & $\mathrm{PFm}$ & 245 & 4.64 & -45 & -49 & 22 \\
\hline & & Left superior frontal gyrus & Fp1 & 61 & 4.11 & -12 & 47 & 28 \\
\hline & & Left superior medial gyrus & & 19 & 3.39 & -9 & 29 & 61 \\
\hline & & Right visual cortex & h0c5 (V5) & 19 & 3.60 & 36 & -73 & 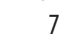 \\
\hline & & Left thalamus & Thal-prefrontal & 21 & 3.04 & -12 & -4 & 13 \\
\hline & & Right amygdala & $C M$ & 20 & 3.86 & 33 & -7 & -17 \\
\hline & & Left cerebellum & IX & 91 & 3.78 & -12 & -55 & -38 \\
\hline & & Right cerebellum & $\mathrm{VI}$ & 10 & 3.32 & 21 & -67 & -20 \\
\hline \multirow[t]{18}{*}{ Right IPS } & \multirow[t]{18}{*}{ ARpar > IRpar } & \multirow[t]{2}{*}{ Right SI } & 1 & 19 & 3.36 & 36 & -34 & 70 \\
\hline & & & 3a & 11 & 3.12 & 27 & -34 & 43 \\
\hline & & \multirow[t]{2}{*}{ Left SII } & $O P 2$ & 17 & 3.67 & -36 & -28 & 25 \\
\hline & & & OP 3 & 14 & 3.58 & -30 & -13 & 16 \\
\hline & & \multirow[t]{2}{*}{ Right SII } & OP1 & 73 & 3.88 & 51 & -16 & 19 \\
\hline & & & $\mathrm{OP2}$ & 51 & 4.02 & 27 & -28 & 25 \\
\hline & & Right insula lobe & 44 & 10 & 3.03 & 48 & 8 & 1 \\
\hline & & Right caudate nucleus & & 117 & 3.79 & 21 & 8 & 16 \\
\hline & & Left caudate nucleus & & 73 & 3.93 & -18 & 14 & 19 \\
\hline & & Right premotor cortex & & 70 & 4.00 & 30 & -4 & 70 \\
\hline & & \multirow[t]{2}{*}{ Left MI } & $4 p$ & 14 & 3.30 & -30 & -16 & 40 \\
\hline & & & $4 a$ & 13 & 3.07 & -21 & -22 & 73 \\
\hline & & Right superior Frontal gyrus & & 63 & 4.07 & 15 & 8 & 46 \\
\hline & & Right visual cortex & hOC1 (V1) & 51 & 3.65 & 30 & -58 & 19 \\
\hline & & Right pallidum & & 11 & 3.02 & 15 & -1 & -5 \\
\hline & & Left thalamus & Thal-prefrontal & 52 & 4.90 & -6 & -16 & -8 \\
\hline & & Right thalamus & Thal-prefrontal & 40 & 3.64 & 15 & -13 & 25 \\
\hline & & Left cerebellum & Lobule VI & 22 & 3.35 & -24 & -67 & -29 \\
\hline \multirow[t]{4}{*}{ Left IPS } & \multirow[t]{4}{*}{ ARpar $>$ IRpar } & Left SII & $0 P 2$ & 19 & 3.36 & -36 & -31 & 22 \\
\hline & & Left MI & $4 a$ & 14 & 3.11 & -15 & -22 & 76 \\
\hline & & Right IPL & PGa & 11 & 3.20 & 51 & -58 & 34 \\
\hline & & Right superior 0ccipital gyrus & & 11 & 3.04 & 24 & -67 & 31 \\
\hline
\end{tabular}

Summary of all calculated PPI analyses ( $p<0.001$, cluster size $k \geq 10$ ). Coordinates are in MNI space. IPL, inferior parietal lobe.

guided spatial attention tasks (Fig. 5). Crucially, the IPS is considered as a central hub embedded in the dorsal frontoparietal network underpinning top-down attentional control (Corbetta and Shulman, 2002), because it has been associated with attentional selection of competing stimuli by calibrating attentional weights (Molenberghs et al., 2007; Vandenberghe et al., 2012). The present connectivity findings suggest that the IPS biases the competition between multiple tactile stimuli by interacting with early somatosensory areas. Although the direction of connectivity cannot directly be concluded from the performed analyses, we hypothesize that the IPS influences somatosensory cortices in a top-down fashion similar to attention-related mechanisms de- scribed for the visual domain (Gillebert et al., 2013). This would also be in-line with a recent EEG-fMRI study on visual spatial attention that revealed an inverse correlation between IPS and posterior alpha activity (Liu et al., 2014), suggesting that IPS modulates visual cortical excitability. In addition to somatosensory cortices, we found strengthened functional coupling between right IPS and (pre)motor areas together with the right caudate nucleus (Fig. 8). Both the (pre)motor cortex and the caudate nucleus play a crucial role in goal-directed actions (Sadato et al., 1997; Grahn et al., 2008). In particular, the premotor cortex is linked to the transformation of sensory information to actions and is thus especially important for perceptual decision 


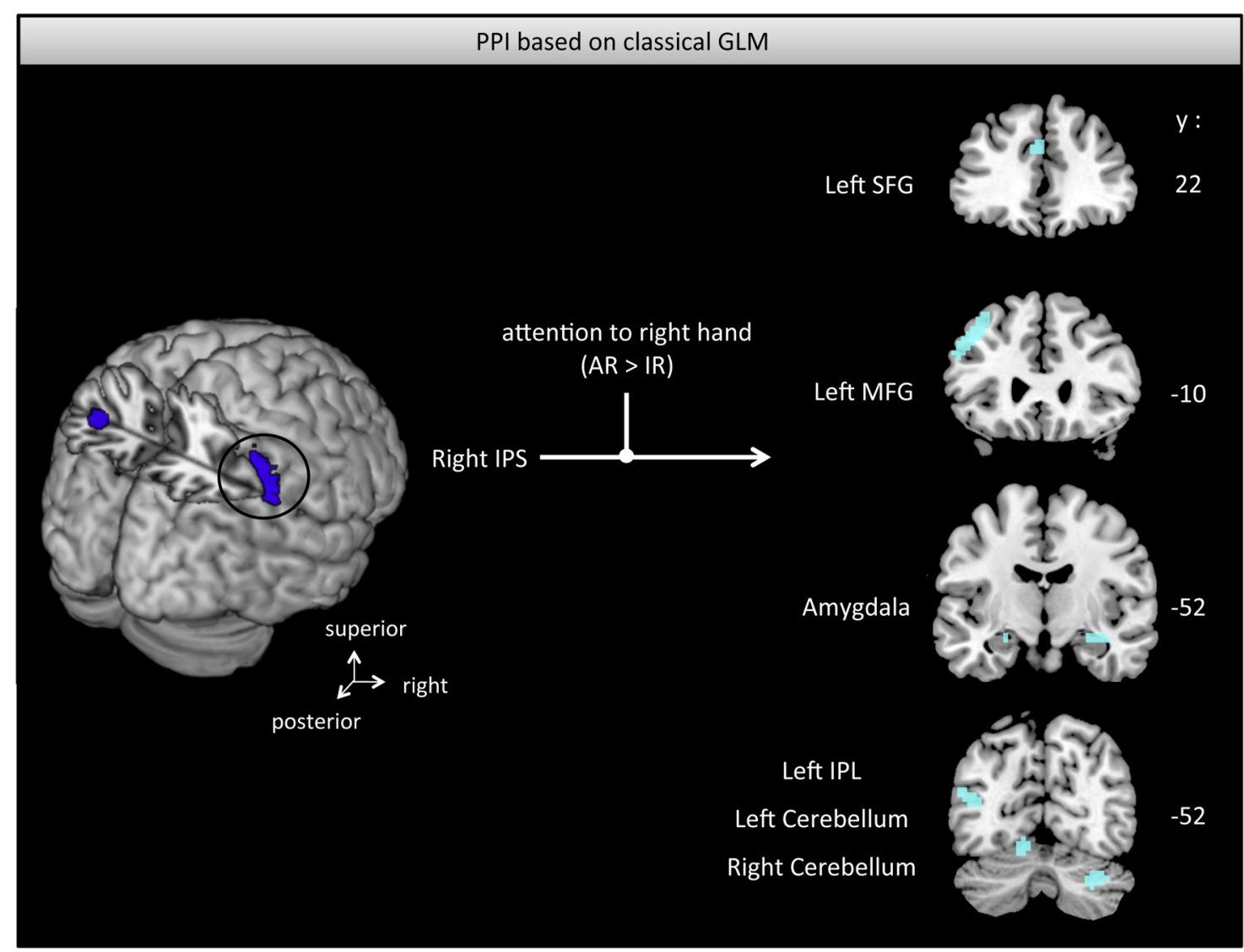

Figure 7. Increased functional connectivity between right IPS and several brain regions (light blue) based on cued attention conditions ( $p<0.005$, uncorrected; classical SSSEP-uninformed GLM). SFG, Superior frontal gyrus; MFG, middle frontal gyrus; IPL, inferior parietal lobe; IPS, intraparietal sulcus. The respective $y$-coordinate is in MNI space.

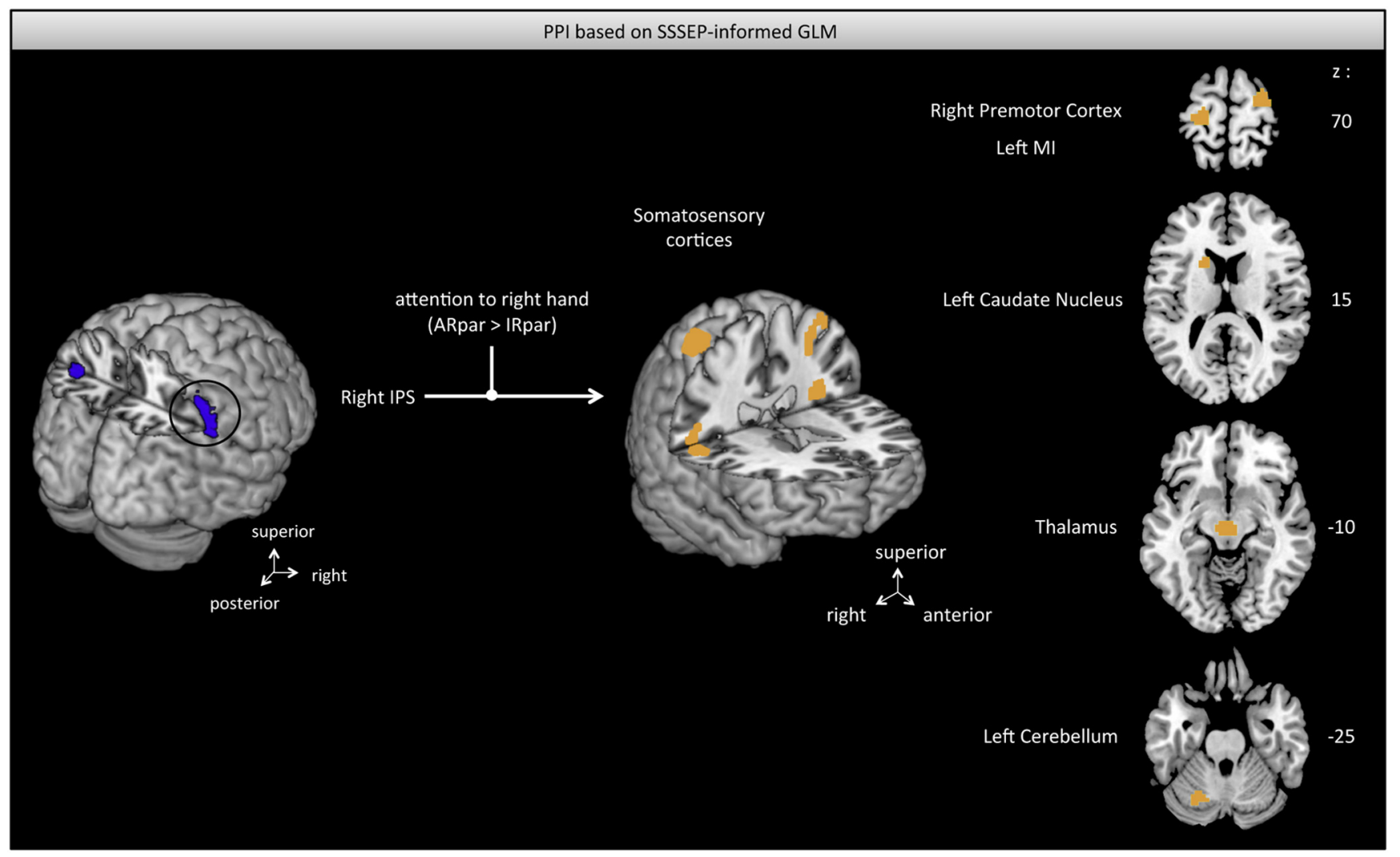

Figure 8. Increased functional connectivity between the right IPS and a sensorimotor network (orange) based on the attention effects provided by SSSEPs ( $p<0.005$, uncorrected). Increased functional coupling was mainly revealed to primary and secondary somatosensory cortex, right premotor and left primary motor cortex, bilateral caudate nucleus, thalamus, and left cerebellum. IPS, Intraparietal sulcus. 
making (Romo et al., 2004; Pastor-Bernier and Cisek, 2011). In addition to attentional processes and executive functioning (Karnath et al., 2002; Hsu et al., 2005), the caudate nucleus also provides important signals to influence and assess perceptual decisions (Platt, 2002; Ding and Gold, 2010, 2013). Because participants' task was to attend to one tactile stream and to indicate whenever they perceived a target, current premotor cortex and caudate nucleus activity may reflect the evaluation of sensory information for the decision to be made. This is in-line with the activity in left primary motor cortex (contralateral to the responding foot) potentially reflecting the preparedness for a button press. As indicated by participants' detection rate of $68.5 \%$, the perceptual decision has been rather difficult presumably causing a strong competition between action choices (i.e., press a button or not). Following that, the increased coupling from IPS to (pre)motor regions and right caudate nucleus may reflect attention-guided selection processes of potential action choices that compete in a sensorimotor map (Pastor-Bernier and Cisek, 2011). This also fits models of somatosensory organization as proposed by Dijkerman and de Haan (2007). Similar to the visual system, they suggested two different processing streams. Whereas one processing stream subserves perception and terminates in the insula, the other stream guides actions and ends in the posterior parietal cortex. With the current study, we provide additional evidence for connections between posterior parietal regions (i.e., the IPS) and action-related sensorimotor areas necessary for translating sensory information into subsequent actions. The influence of IPS activity on motor actions is further corroborated by the significant relationship between SSSEPs and subsequent behavioral performance. On the one hand, we found significantly lower steady-state amplitudes before missed target events than before hits. On the other hand, we found that the higher the steady-state responses, the faster were the reaction times to correctly detected targets in upcoming trials. Given that SSSEP values significantly correlated with IPS activity, it seems as if IPS activity triggered forthcoming behavioral performance. Thus, based on previous literature and in light of the present findings, we propose that the right IPS is not only involved in the attention-related selection process between competing tactile stimuli, but also in referencing sensory information to potential actions by selecting between action choices.

Given that we compared left and right attention conditions, our study is comparable to previous work on lateralized attention and motor preparation. Especially, the relationship between steady-state amplitude and upcoming behavioral performance shows considerable parallels to the work on prestimulus oscillations: for example, visual and tactile spatial attention studies showed that decreases in alpha and beta power were associated with better discrimination performance and faster reaction times to subsequently presented stimuli (van Dijk et al., 2008; Haegens et al., 2011; van Ede et al., 2011). Moreover, Mazaheri et al. (2009) showed that increased occipital alpha and sensorimotor mu activity predicted behavioral errors before they actually occur. However, although these findings point to a negative relationship between prestimulus oscillatory activity and performance (i.e., with lower-power better performance or vice versa), our results indicate a positive relationship (with lower amplitude worse behavioral performance; Fig. 3). This difference is most likely caused by the different nature of oscillatory signals. Whereas studies on prestimulus activity measured spontaneous internal oscillations and spatial attention led to decreased amplitudes in neural populations processing stimuli in the attended location (Jones et al., 2010), we measured externally driven SSSEPs which showed increased amplitudes with attention. Moreover, we applied tactile stimulation in the beta frequency range that was associated to influence performance in touch (van Ede et al., 2011) and were interested in a time window that was temporally more distant to the behavioral responses. Nonetheless, our findings corroborate a close relationship between oscillatory activity and subsequent performance and highlight the significant impact of prestimulus activity.

An open question may still be why we only found a significant activation for the right and not for the left finger stimulation. In general, one should highlight that the right IPS was also revealed by trend for the left finger. As participants' detection rate was significantly higher for the right stimulation, one explanation is that it was easier for participants to attend to the right hand. However, given that all our subjects were right-handed, we cannot infer whether this is unique to the right or the dominant hand. Future research is needed to disentangle different activation patterns for the right and left finger in face of hand dominance.

Overall, the current study demonstrates that classical analyses do not capture fast fluctuating cognitive states and associated network function, and thus conceals valuable information. A reliable control of single-trial attentional modulation as provided by simultaneous recordings of SSSEPs during fMRI, however, will help to create a more fine-grained picture of processes underpinning attentional mechanisms during sustained attention.

\section{References}

Behrmann M, Geng JJ, Shomstein S (2004) Parietal cortex and attention. Curr Opin Neurobiol 14:212-217. CrossRef Medline

Burton H, Sinclair RJ, McLaren DG (2008) Cortical network for vibrotactile attention: a fMRI study. Hum Brain Mapp 29:207-221. CrossRef Medline

Corbetta M, Shulman GL (2002) Control of goal-directed and stimulusdriven attention in the brain. Nat Rev Neurosci 3:201-215. Medline

Desimone R, Duncan J (1995) Neural mechanisms of selective visualattention. Annu Rev Neurosci 18:193-222. CrossRef Medline

Dijkerman HC, de Haan EH (2007) Somatosensory processes subserving perception and action. Behav Brain Sci 30:189-201; discussion 201-39. CrossRef Medline

Ding L, Gold JI (2010) Caudate encodes multiple computations for perceptual decisions. J Neurosci 30:15747-15759. CrossRef Medline

Ding L, Gold JI (2013) The basal ganglia's contributions to perceptual decision making. Neuron 79:640-649. CrossRef Medline

Eickhoff SB, Paus T, Caspers S, Grosbras MH, Evans AC, Zilles K, Amunts K (2007) Assignment of functional activations to probabilistic cytoarchitectonic areas revisited. Neuroimage 36:511-521. CrossRef Medline

Friston KJ, Buechel C, Fink GR, Morris J, Rolls E, Dolan RJ (1997) Psychophysiological and modulatory interactions in neuroimaging. Neuroimage 6:218-229. CrossRef Medline

Galazky I, Schütze H, Noesselt T, Hopf JM, Heinze HJ, Schoenfeld MA (2009) Attention to somatosensory events is directly linked to the preparation for action. J Neurol Sci 279:93-98. CrossRef Medline

Giabbiconi CM, Dancer C, Zopf R, Gruber T, Müller MM (2004) Selective spatial attention to left or right hand flutter sensation modulates the steady-state somatosensory evoked potential. Brain Res Cogn Brain Res 20:58-66. CrossRef Medline

Giabbiconi CM, Trujillo-Barreto NJ, Gruber T, Müller MM (2007) Sustained spatial attention to vibration is mediated in primary somatosensory cortex. Neuroimage 35:255-262. CrossRef Medline

Gillebert CR, Caspari N, Wagemans J, Peeters R, Dupont P, Vandenberghe R (2013) Spatial stimulus configuration and attentional selection: extrastriate and superior parietal interactions. Cereb Cortex 23:2840-2854. CrossRef Medline

Goltz D, Pleger B, Thiel S, Villringer A, Müller MM (2013) Sustained spatial attention to vibrotactile stimulation in the flutter range: relevant brain regions and their interaction. PLoS One 8:e84196. CrossRef Medline

Grahn JA, Parkinson JA, Owen AM (2008) The cognitive functions of the caudate nucleus. Prog Neurobiol 86:141-155. CrossRef Medline 
Gundlach C, Müller MM (2013) Perception of illusory contours forms intermodulation responses of steady state visual evoked potentials as a neural signature of spatial integration. Biol Psychol 94:55-60. CrossRef Medline

Haegens S, Nácher V, Luna R, Romo R, Jensen O (2011) $\alpha$-Oscillations in the monkey sensorimotor network influence discrimination performance by rhythmical inhibition of neuronal spiking. Proc Natl Acad Sci U S A 108:19377-19382. CrossRef Medline

Hsu M, Bhatt M, Adolphs R, Tranel D, Camerer CF (2005) Neural systems responding to degrees of uncertainty in human decision-making. Science 310:1680-1683. CrossRef Medline

Johansen-Berg H, Christensen V, Woolrich M, Matthews PM (2000) Attention to touch modulates activity in both primary and secondary somatosensory areas. Neuroreport 11:1237-1241. CrossRef Medline

Jones SR, Kerr CE, Wan Q, Pritchett DL, Hämäläinen M, Moore CI (2010) Cued spatial attention drives functionally relevant modulation of the mu rhythm in primary somatosensory cortex. J Neurosci 30:13760-13765. CrossRef Medline

Karnath HO, Himmelbach M, Rorden C (2002) The subcortical anatomy of human spatial neglect: putamen, caudate nucleus and pulvinar. Brain 125:350-360. CrossRef Medline

Liu Y, Bengson JJ, Huang H, Mangun GR, Ding M (2014) Top-down modulation of neural activity in anticipatory visual attention: control mechanisms revealed by simultaneous EEG-fMRI. Cereb Cortex. Advance online publication. doi:10.1093/cercor/bhu204. CrossRef Medline

Liu Z, de Zwart JA, van Gelderen P, Kuo LW, Duyn JH (2012) Statistical feature extraction for artifact removal from concurrent fMRI-EEG recordings. Neuroimage 59:2073-2087. CrossRef Medline

Mayer AR, Dorflinger JM, Rao SM, Seidenberg M (2004) Neural networks underlying endogenous and exogenous visual-spatial orienting. Neuroimage 23:534-541. CrossRef Medline

Mazaheri A, Nieuwenhuis IL, van Dijk H, Jensen O (2009) Prestimulus alpha and mu activity predicts failure to inhibit motor responses. Hum Brain Mapp 30:1791-1800. CrossRef Medline

Molenberghs P, Mesulam MM, Peeters R, Vandenberghe RR (2007) Remapping attentional priorities: differential contribution of superior parietal lobule and intraparietal sulcus. Cereb Cortex 17:2703-2712. CrossRef Medline

Morgan ST, Hansen JC, Hillyard SA (1996) Selective attention to stimulus location modulates the steady-state visual evoked potential. Proc Natl Acad Sci U S A 93:4770-4774. CrossRef Medline

Nierhaus T, Gundlach C, Goltz D, Thiel SD, Pleger B, Villringer A (2013) Internal ventilation system of MR scanners induces specific EEG artifact during simultaneous EEG-fMRI. Neuroimage 74:70-76. CrossRef Medline
Oldfield RC (1971) The assessment and analysis of handedness: the Edinburgh inventory. Neuropsychologia 9:97-113. CrossRef Medline

Pang CY, Mueller MM (2014) Test-retest reliability of concurrently recorded steady-state and somatosensory evoked potentials in somatosensory sustained spatial attention. Biol Psychol 100:86-96. CrossRef Medline

Pastor-Bernier A, Cisek P (2011) Neural correlates of biased competition in premotor cortex. J Neurosci 31:7083-7088. CrossRef Medline

Platt ML (2002) Neural correlates of decisions. Curr Opin Neurobiol 12: 141-148. CrossRef Medline

Ritter P, Villringer A (2006) Simultaneous EEG-fMRI. Neurosci Biobehav Rev 30:823-838. CrossRef Medline

Romo R, Hernández A, Zainos A (2004) Neuronal correlates of a perceptual decision in ventral premotor cortex. Neuron 41:165-173. CrossRef Medline

Sadato N, Yonekura Y, Waki A, Yamada H, Ishii Y (1997) Role of the supplementary motor area and the right premotor cortex in the coordination of bimanual finger movements. J Neurosci 17:9667-9674. Medline

Severens M, Farquhar J, Duysens J, Desain P (2013) A multi-signature brain-computer interface: use of transient and steady-state responses. J Neural Eng 10:026005. CrossRef Medline

Snyder AZ (1992) Steady-state vibration evoked potentials: description of technique and characterization of responses. Electroencephalogr Clin Neurophysiol 84:257-268. CrossRef Medline

Tipper SP, Weaver B, Houghton G (1994) Behavioral goals determine inhibitory mechanisms of selective attention. Q J Exp Psychol A 47:809840. CrossRef

van Dijk H, Schoffelen JM, Oostenveld R, Jensen O (2008) Prestimulus oscillatory activity in the alpha band predicts visual discrimination ability. J Neurosci 28:1816-1823. CrossRef Medline

van Ede F, de Lange F, Jensen O, Maris E (2011) Orienting attention to an upcoming tactile event involves a spatially and temporally specific modulation of sensorimotor alpha- and beta-band oscillations. J Neurosci 31:2016-2024. CrossRef Medline

Vandenberghe R, Geeraerts S, Molenberghs P, Lafosse C, Vandenbulcke M, Peeters K, Peeters R, Van Hecke P, Orban GA (2005) Attentional responses to unattended stimuli in human parietal cortex. Brain 128:28432857. CrossRef Medline

Vandenberghe R, Molenberghs P, Gillebert CR (2012) Spatial attention deficits in humans: the critical role of superior compared with inferior parietal lesions. Neuropsychologia 50:1092-1103. CrossRef Medline

Zopf R, Giabbiconi CM, Gruber T, Müller MM (2004) Attentional modulation of the human somatosensory evoked potential in a trial-by-trial spatial cueing and sustained spatial attention task measured with high density 128 channels EEG. Brain Res Cogn Brain Res 20:491-509. CrossRef Medline 